\begin{tabular}{lllllllllllllllllllllllllllllllll}
\hline$R$ & $E$ & $V$ & I & S & T & A & D & E & E & S & T & U & D & I & O & S & I & N & T & E & R & N & A & C & I & O & N & A & L & E & S
\end{tabular}

\title{
Las percepciones de amenazas y de seguridad entre Europa y el mundo árabe*
}

\author{
Martín Jerch y Alejandro V. Lorca Corróns
}

La Unión Europea ha declarado 2005 año del Mediterráneo, por ser el décimo aniversario del llamado Proceso de Barcelona, la política de la Unión Europea frente al los países de la cuenca sur del Mediterráneo. Es la primera vez que la UE incluye aspectos no solo económicos sino también de seguridad, de política y temas humanitarios y sociales en su política para la región. Este artículo analiza uno de los aspectos más esenciales de la seguridad en el Mediterráneo: las percepciones de amenaza. Se argumenta que su identificación se vincula estrechamente con una serie de factores subjetivos y que una de las principales diferencias en las percepciones de amenaza reside en los diferentes grados de desarrollo, tanto económicos como políticos, que existen entre ambas orillas del Mediterráneo. Aplicando este concepto de seguridad se estudian las amenazas que se percibe a cada lado de la cuenca mediterránea. Una vez determinadas las amenazas y los riesgos, se examina la viabilidad, es decir, de las amenazas enumeradas. Se concluye que ella es muy baja $y$, debe explicarse por las considerables diferencias entre ambas orillas, diferencias que en la última parte del trabajo se atribuyen a la dicotomía entre "nosotros" y "ellos".

Este artículo es una versión modificada del artículo "Seguridad y Percepciones en el Mediterráneo" publicado en la revista Estudios Político Militares, Vol. 4, N ${ }^{\circ}$ 7, 2004, pp. 5-81. 


\section{INTRODUCCIÓN}

a seguridad en el Mediterráneo es un tema complejo e introducir al lector en esta complejidad no resulta sencillo. No obstante, es necesario abordarlo, pues el Mediterráneo a menudo ha producido y producirá dolores de cabeza a los responsables de la política exterior europea. De hecho, hay abundantes artículos y libros que tratan desde diferentes perspectivas el tema de la seguridad en el Mediterráneo. La principal pregunta que se hacen los autores es lo que comprende el concepto «seguridad», en general, y seguridad en el Mediterráneo, en particular. Seguridad es un término que se utiliza a diario, en diferentes ámbitos; un término cuyo significado en cierta medida todos conocemos, pero acerca del cual pocas veces se reflexiona en profundidad. Desde la seguridad alimentaria hasta la seguridad vial, el término seguridad se puede combinar con miles de atributos y siempre se consigue un nuevo concepto. Si nos acercamos a los estudios de seguridad a nivel político internacional, el término también ha evolucionado y la complejidad de las interrelaciones hace cada vez más difícil su definición. Desde la seguridad nacional hasta la seguridad ecológica, todo es posible.

Por tanto, es importante definir el concepto «seguridad». Desde una perspectiva global, los estudios sobre la seguridad internacional son una importante subdisciplina del estudio de las relaciones internacionales, guiada por la pregunta de ¿cómo se puede aumentar la seguridad internacional? Incluso puede afirmarse que los estudios sobre la seguridad a nivel internacional son la piedra angular, la razón de ser de las relaciones internacionales.

\section{Los estudios sobre la seguridad son la piedra angular de las relaciones internacionales.}

La inesperada desaparición del muro de Berlín, símbolo del conflicto bipolar entre Este y Oeste, ha causado un enorme cambio en las relaciones internacionales. Este conflicto, que dominó los estudios desde el final de la Segunda Guerra Mundial, ha producido una considerable cantidad de trabajos. Y con la desaparición del conflicto, parece que todo lo elaborado hasta 1990 pierde validez. Sin embargo, hay una serie de estudios acerca del problema de las percepciones llevados a cabo en el contexto de la confrontación bipolar por psicólogos y sociólogos, que introduciremos aquí para sensibilizar al lector respecto de los problemas relacionados con la seguridad en el Mediterráneo. No obstante, los terribles atentados terroristas del 11 de septiembre de 2001 en Nueva York y el 11 de marzo de 2004 aquí, en Madrid, han dado una nueva dimensión al concepto de seguridad. En efecto, cada vez más autores vinculan la seguridad internacional con el desarrollo, o sea, argumentan que el origen de una serie de amenazas se encuentra en la falta de desarrollo económico y político.

El primer objetivo es sensibilizar al público acerca de la complejidad de los problemas que existen en las relaciones de Europa con sus vecinos de la cuenca 
sur del Mediterráneo. Hasta ahora no se ha hecho mucho por informar de forma objetiva sobre los problemas del Mediterráneo, sin duda porque ellos son poco conocidos y peor entendidos. Al menos hasta hace muy poco, la Unión Europea y Estados Unidos en buena medida han optado por ignorar al Mediterráneo. El 11-S y la lucha contra el terrorismo parecen haber despertado el interés por el Mediterráneo y el Oriente Medio. Y a pesar de que 2004 estuvo lleno de nuevas iniciativas para aumentar la seguridad, la mayoría de ellas desaparecerá con la misma rapidez con que aparecieron, en gran medida porque no tratan adecuadamente los problemas de la zona. Tomar la seguridad como algo subjetivo, en cuya base se encuentra la percepción, un proceso cognitivo, hace que nuestro enfoque del Mediterráneo se diferencie de la mayoría de los trabajos sobre la seguridad realizados hasta ahora. Por ello, nuestro segundo objetivo consiste precisamente en analizar los factores que quizás han influido o influyen en las percepciones de ambas orillas.

Con el fin de la Guerra Fría ha aumentado el interés de los estudiosos occidentales por la región mediterránea, sobre todo por las relaciones con los países árabes que se encuentran en su ribera sur. Los acontecimientos del 11 de septiembre de 2001 han dado la razón a los expertos que detectaron un embrión de conflictos en la inestabilidad interna por la que atraviesa el mundo árabe. En la discusión estratégica de los primeros años tras la caída del Muro de Berlín, a menudo se podía leer acerca de una «nueva amenaza» proveniente del sur (Fisas, 1991; Cosidó, 1995). Para las percepciones europeas, estas amenazas residen en primer lugar en la región árabe-musulmana de la cuenca sur del Mediterráneo. El poco afortunado y antiguo secretario general de la Alianza Atlántica, el belga Willy Claes, afirmó en una entrevista que «el fundamentalismo es casi tan peligroso como fue el comunismo» (cit. en Cosidó, 1995:167) ${ }^{1}$.

\section{La inestabilidad interna del mundo árabe es un embrión de conflictos.}

En el ámbito académico, el estadounidense Samuel P. Huntington (1996) proclamó el «choque de civilizaciones». De acuerdo con este autor, los conflictos del futuro se darán entre diferentes civilizaciones, de las cuales la islámica es la más conflictiva, y se producirán choques entre el mundo islámico y las civilizaciones que tienen fronteras con él. Desafortunadamente, el atentado terrorista contra las Torres Gemelas y el Pentágono y la «declaración de guerra» por parte del presidente George W. Bush parece, en un primer momento, dar la razón a la visión de Huntington. A los pocos días de este atentado la Casa Blanca se apresuró en aclarar que la guerra no era contra el Islam sino contra el terrorismo. En Occidente todo responsable político insiste en este punto, aunque su interpretación no llega

1 Entrevista publicada el 2 de febrero 1995 en el periódico alemán Süddeutsche Zeitung. 
con claridad a las calles de las ciudades islámicas, donde los ciudadanos más activos lo interpretan como una confrontación contra el Islam. Parece que todo se ha vuelto contra los países árabes. Por ello, es necesario dar una visión más sofisticada de los problemas que existen en el Mediterráneo y de las relaciones entre Europa y los denominados Países Mediterráneos No Comunitarios $(\mathrm{PMNC})^{2}$, a fin de eximirlos del «excepcionalismo» con que a menudo se simplifican en exceso los problemas de la región y sus relaciones con la UE y Estados Unidos.

\section{Todo parece haberse vuelto contra los países árabes.}

Para alcanzar nuestro objetivo, hemos estructurado nuestro trabajo como sigue. En primer lugar, se ofrece una breve discusión general sobre el término «seguridad», para ayudar al lector a entender mejor un concepto repleto de subjetividad. Tras esta discusión, más bien teórica, en el segundo capítulo se señalan las diferentes amenazas y riesgos percibidos por los actores de la región. Luego de esta parte empírica se analiza la relación entre desarrollo y seguridad, que a nuestro juicio es esencial para poder entender las relaciones internacionales del siglo XXI. A continuación, se estudian los factores que podrían influir en las percepciones antes identificadas.

$$
\text { ¿QUÉ ES SEGURIDAD? }
$$

Si se busca una enciclopedia cualquiera, se encontrará una descripción parecida a la de Franz X. Kaufmann (1973), para quien el término «seguridad» tiene un elemento de «carencia de peligro». Asegurar significa «proteger del peligro». Este autor destaca que de ser un símbolo lingüístico la noción de «seguridad» pasó a símbolo de valor social. Si nos acercamos más a los trabajos teóricos sobre seguridad, observamos que casi cada contribución ofrece una definición diferente. Una definición que nos acerca más a la idea de la subjetividad es la de Daase (1993), para quien «seguridad es lo que un actor político o una unidad política llama en una situación determinada seguridad»o, en otras palabras «la política de seguridad determina quién define la amenaza». Esta definición incluye el aspecto subjetivo de la seguridad.

Toda la información o desinformación que recibimos y que influye en nuestros sentimientos de seguridad o inseguridad se transmite a través de un proceso de comunicación. Por ejemplo, si no hubiéramos estado a punto de viajar en el tren de cercanía volado por los terroristas el 11 de marzo de 2004 en la estación de Atocha, ahora no sentiríamos cierto miedo cuando viajamos en estos trenes. Por tanto, concebimos esencialmente la percepción de seguridad o de amenaza como un proceso de comunicación, donde un actor transmite señales y otro actor las

2 Término usado por la Unión Europea para referirse al conjunto de países que incluye Argelia, Egipto, Israel, Jordania, Líbano, Marruecos, Siria, Túnez y Turquía. También incluye como actor no estatal a la Autoridad Palestina. 
recibe y las interpreta de acuerdo con sus sentimientos.

En un documento de la UNESCO se afirma que «las guerras comienzan en las mentes de los hombres». Dicho de otro modo, el principal origen de los conflictos $\mathrm{y}$ de sentirse seguro o inseguro son las percepciones humanas (Piris, 2001:15). Basándose en este fenómeno, se puede afirmar que la inseguridad deriva de la interpretación y de la contemplación subjetiva recíproca de los actores de un sistema acerca de sus necesidades de seguridad, sus ambiciones y objetivos. La seguridad como modelo de relación entre Estados se determina por el grado de necesidad y el grado de amenaza. Ambos factores se transmiten a través de un proceso de comunicación. Idealmente, un proceso de comunicación supone que el mensaje enviado por una parte es recibido por la otra en la misma forma en que fue enviado.

Hay toda una serie de factores que influyen en la forma de enviar y recibir un mensaje, de manera que el proceso de comunicación ideal puede ser distorsionado por elementos que afectan la recepción de los mensajes. Se trata de un proceso que se da a menudo en nuestra vida cotidiana. El estímulo o mensaje que fluye entre dos o más participantes en un proceso de comunicación está influido por una serie de factores tanto externos como internos. Todos estos factores determinan la percepción y la sensación de seguridad o inseguridad. Este ejemplo a nivel de comunicación personal se puede aplicar por analogía a las relaciones entre Estados. La forma en que un actor reacciona frente a una amenaza depende de la valoración que él realice de dicha amenaza; es decir, depende de la «definición de la situación» por el actor, tal y como este la está percibiendo (Gantzel, 1972). La vinculación entre la amenaza existente y la percepción e interpretación de cada uno de los actores son muy importante. El teorema de Thomas expresa correctamente esta relación al señalar que: «Cuando el hombre define una situación como real, esta situación es real en sus consecuencias» (Mertas, 1957: 421; cit. en Gantzel, 1972: 121$)^{3}$.

\section{Las percepciones humanas son el origen de los conflictos y de la sensación de seguridad o inseguridad.}

Según Singer (1958: 93), la «definición de la situación», que es el punto inicial de cada percepción de amenaza, se determina, fundamentalmente por: a) las condiciones objetivas en que debe actuar un actor y b) las predisposiciones (preexisting attitudes), es decir, los juicios y las actitudes. Si la percepción de la amenaza depende de la capacidad de amenazar y de los supuestos propósitos del contrario, como lo supone Singer (1958: 94), la seguridad depende tanto de la necesidad de seguridad de cada uno de los actores como de la transmisión de las percepciones de amenaza (Schölch 1989 :109). En el proceso de comunicación influyen

3 Todas las traducciones fueron realizadas por los autores. 
malentendidos y estimaciones falsas, debido a que los actores tienden a interpretar las señales y el comportamiento de los demás según sus propias predisposiciones (Jervis 1976: 205). Basándose en esta argumentación teórica, en el siguiente capítulo se analizan las percepciones de amenaza y de riesgo en el Mediterráneo.

\section{El Mediterráneo se ha convertido en centro de atención de políticos y de expertos.}

\section{LAS PERCEPCIONES DE AMENAZA Y DE RIESGO EN EL MEDITERRÁNEO}

A raíz de la caída del Muro de Berlín, se publicaron numerosos estudios acerca de la nueva dimensión de la seguridad en el Mediterráneo, con énfasis en la dimensión no militar (Aliboni etal., 1996; Khalilzad et al., 1998; Larrabee et al., 1998; Lesser, 2000). El Mediterráneo se ha convertido -sin duda por el conflicto árabe-israelí y por el problema del abastecimiento de petróleo- en centro de atención de políticos y expertos. La agenda de seguridad en el Mediterráneo es una de las más complejas del mundo y la lista de amenazas y riesgos incluye desde temas de «seguridad dura» (hard security), como por ejemplo la proliferación de armas de destrucción masiva, hasta asuntos que generalmente se agrupan bajo el título de «seguridad blanda» (soft security), que tiene sus raíces en cuestiones económicas, políticas y sociales (nar- cotráfico, redes de inmigración ilegal e inestabilidad política en los países de sur).

En términos militares los problemas de seguridad siguen teniendo cierta importancia en la región y parecen haber recibido un importante impulso con la estrategia estadounidense de guerra preventiva y el hecho de que la mayor parte de los declarados enemigos de Washington se encuentran en la zona. Esta dimensión militar se extiende desde la violencia política y el terrorismo hasta la proliferación de armas de destrucción masiva y de misiles balísticos de largo alcance, sin excluir las confrontaciones armadas entre los Estados del sur. Además, existen considerables arsenales de armas convencionales, que pueden representar un desafío a la integridad territorial de los países vecinos. Sin embargo, los riesgos militares directos se limitan más bien a la dimensión sursur.

Quizás una remota posibilidad sería una crisis hispano-marroquí en torno a los enclaves españoles de Ceuta y Melilla, o crisis como la del islote de Perejil, en 2002. Desde el punto de vista del sur, existe la percepción de amenaza militar en forma de intervención por el norte en los asuntos internos, principio que se incorporó en la Declaración de Barcelona debido a las exigencias de los PMNC. Estas percepciones se nutren en el nuevo concepto adoptado por la OTAN en Roma en 1991 y en maniobras en el contexto de Dragon Hammer que, ante todo en Argelia, fueron percibidas como riesgo de una posible intervención en los asuntos internos (Biad, 1998). De manera similar, Chourou (1998) señala que los PMNC no representan nin- 
gún riesgo militar para el norte debido a su falta de capacidad militar pero, en cambio, dichos países tienen que vivir con la constante posibilidad de ser blanco de operaciones militares europeas. El mismo autor detecta que a pesar del fin de la Guerra Fría y de la desaparición de la amenaza comunista, la capacidad militar europea $^{4}$ no ha disminuido de manera manifiesta. El sur percibe una amenaza de intervención del Norte en sus asuntos internos que nunca se ha materializado de manera directa, aunque sí indirectamente, como en el caso de Argelia, aunque con el consentimiento del gobierno argelino. En realidad, en el sur siempre se percibe que la amenaza militar proviene de los países árabes vecinos.

\section{Los riesgos militares directos se limitan más bien a la dimensión sur-sur.}

A los riesgos y las amenazas en el sentido «clásico» aquí señalados se suman otros que constituyen la nueva agenda de seguridad en el contexto del Mediterráneo. Muchos asocian esta dimensión con el denominado conflicto norte-sur. Es decir, el conflicto entre el primero, industria- lizado y rico, y el segundo, subdesarrollado y pobre, en contraposición al conflicto este-oeste, que se centró ante todo en la dimensión militar. Así, Bichara Khader habla del conflicto norte-sur y de la «militarización del propio concepto de seguridad» (1994: 284). En el debate estratégico actual se reconoce ampliamente que los problemas de la seguridad blanda obedecen a la creciente diferencia entre los niveles de desarrollo de ambas orillas del Mediterráneo, considerada fuente de inestabilidad en la región.

De hecho, la mayoría de los expertos europeos y estadounidenses consideran la seguridad en el Mediterráneo en términos de estabilidad, y utilizan los términos «seguridad»y «estabilidad» como sinónimos. El primer Libro Blanco de la Defensa 2000 español, por ejemplo, indica que «el Mediterráneo es un área de contraste y desequilibrios en los órdenes político, económico y social y, en consecuencia, propenso a la inestabilidad.» (p. 33).

La estabilidad en la región mediterránea es, en primer lugar, una cuestión de política interna con posibles efectos de desbordamiento en toda la región. En el orden político interior influyen aspectos económicos, demográficos y sociales. Muchos de los PMNC carecen de legiti-

4 Fisas (1991) compara el potencial militar entre ambas orillas del Mediterráneo occidental y llega a la conclusión de que, según las capacidades existentes, es el norte que amenaza al sur, y no al revés. Esta argumentación, que se apoya en las impresionantes diferencias en términos militares, subraya a su vez el punto de vista de los países del sur. Sin embargo, este análisis y su significado explicativo deben tomarse con cautela, porque Fisas ignora por completo las contribuciones de estudios como los de Robert Jervis (1976), que han determinado una serie de factores de percepción. Es decir, no solo influyen las cifras absolutas, sino una serie de factores. Aplicando la comparación a Alemania y Luxemburgo, resulta fácil imaginar diferencias muy parecidas; sin embargo, actualmente nadie se sentiría amenazado por Alemania. 
midad política, lo que afecta la capacidad de tomar decisiones para resolver los problemas de estabilidad. En los temas de seguridad, la Declaración de Barcelona introdujo esta nueva dimensión de estabilidad y con ella una nueva interrelación entre las relaciones internacionales y las políticas internas que puede causar tensiones entre los PMNC y la UE. Con creciente frecuencia se apunta más a las tensiones internas como origen de la percepción de amenazas en el Mediterráneo. El problema de identificar estabilidad con seguridad en forma simplista es que puede significar mantener el statu quo político y económico de los países del sur. Por definición, la transición de regímenes políticos autoritarios a otros más abiertos y la liberalización de economías intervenidas y patrimoniales generan luchas políticas internas y, por tanto, inestabilidad a corto plazo. Pero al mismo tiempo, mantener esos sistemas político-económicos nutre la inestabilidad a largo plazo. Este dilema entre estabilidad a corto y largo plazos es el núcleo del problema de seguridad en la zona sur del Mediterráneo.

\section{El cambio de políticas emanado del consenso de Washington ha tenido altos costes sociales para los países de la región.}

La cuestión de la legitimidad de los gobiernos para realizar las reformas se relaciona directamente con factores económicos y demográficos. En lo que toca a los aspectos económicos, destacan ante todo las consecuencias sociales de las reformas y de la liberalización económica de la última década. La necesidad de reformas es el resultado de un largo período de mala gestión que se acompañó de la creación de un aparato burocrático sobredimensionado, donde la corrupción y el soborno eran prácticas habituales. El cambio de políticas de sustitución de importaciones a políticas de apertura de las economías, de acuerdo con la posición del "consenso de Washington", ha tenido elevados costes sociales para estos países. Gracias a los ingresos del petróleo (en los países que lo producen), se podía calmar y satisfacer a la población. Pero con un crecimiento demográfico excesivo no se puede mantener un crecimiento económico sostenido. Además, son pocos los Estados productores de petróleo y sus ingresos son cíclicos, ya que los precios oscilan frecuentemente. En consecuencia, en este nuevo contexto los que más sufren son los que desde siempre han tenido nada o muy poco. Por el contrario, la nueva clase de empresarios es la que más se beneficia con las reformas económicas. El aumento de las diferencias entre ricos y pobres hace que estas sociedades sean inestables. Como consecuencia de esta situación económica -unida a la marginación de las zonas rurales- las sociedades de los PMNC registran altas tasas de urbanización, las más altas de todo el mundo (Larrabee et al., 1998: 8). Por su parte, la urbanización es un factor importante para la estabilidad de los regímenes políticos, ya que la migración rural destruye los patrones de comportamiento tradicionales y da lugar a nuevas demandas a los gobier- 
nos, en forma de empleo, viviendas y escuelas (Farrar-Hockley, 1994: 72).

La incapacidad de los responsables políticos de hacer frente a estas demandas es la causa del incremento de la población urbana que organiza su vida de manera ajena al Estado, buscando sus referencias y apoyos en los movimientos islámicos que poseen una logística y una organización municipal eficientes ${ }^{5}$. Este mecanismo es una de las soluciones perseguidas por los ciudadanos que se sienten abandonados por el poder y la elite política. Otra alternativa es salir del país y buscar su fortuna en los países europeos. De esta forma, el fracaso económico de la elite se transforma en inestabilidad social con la introducción y desarrollo de un movimiento islamista que sustituye a un Estado ausente en los servicios sociales, médicos y de enseñanza en las zonas marginales urbanas. Este movimiento islámico de raíces populares introduce inestabilidad política y pone en duda la legitimidad del gobierno de la elite dominante. El conflicto es claramente interno, pero al desbordarse crea un conflicto internacional: surge un enfrentamiento entre las formas europeas de la elite y los comportamientos islámicos de los movimientos populares. Además, el apoyo a los movimientos islamistas es utilizado para debilitar a los gobiernos vecinos, con los que no siempre se mantienen buenas relaciones.

En suma, los riesgos se presentan ante todo en forma de movimientos sociopolíticos radicales, que se relacionan con los movimientos islamistas y la migración ilegal hacia Europa, que es percibida como un desafío a la seguridad interna por los países europeos. Las causas de estos dos fenómenos son en gran media internas, de origen político y económico, pero sus repercusiones son regionales o incluso transregionales.

\section{El movimiento islamista sustituye al Estado en la satisfacción de las necesidades de las zonas marginales urbanas.}

Los movimientos migratorios y la creciente movilización política radical islamista (a su vez vinculados con la migración y el terrorismo) son los dos elementos claves en torno a los cuales se centran los estudios sobre las amenazas no militares. En los últimos decenios, conflictos como los de Argelia, Sudán y, sobre todo, el de Afganistán y los conflictos en los Balcanes, han dado origen a grupos violentos entrenados en estas guerras, bien organizados y financiados, como el Grupo Al-Qaeda, responsable de los actos terroristas del 11 de septiembre. Este terrorismo internacional es percibido como una amenaza dura por occidente, que utiliza todos los mecanismos para contrarrestar la amenaza militar, incluyendo la guerra preventiva.

Los movimientos islamistas representan una alternativa al déficit en asuntos sociales del Estado, ofreciendo servicios de sanidad, educación, formación de los jóvenes, y así se ha creado un tipo de «contra-sociedad», que se basa en redes de mezquitas en las que se vive estrictamente según las normas del Corán (ver Blunden 1994: 142). 
Tanto los cambios políticos como la nueva situación geoestratégica afectan al Mediterráneo en diferentes formas: desde la revolución islámica en Irán, pero sobre todo tras la victoria de un partido islamista en Argelia, se considera al Islam político violento como actor importante en los desafíos internos y externos. No se puede negar la importancia del Islam político como factor que determina las relaciones internas y externas de estos países. Pero no debe olvidarse ni subestimarse el potencial desestabilizador del nacionalismo en el Mediterráneo: Grecia, Turquía, Siria, etc. son buenos ejemplos de ello.

Un fenómeno muy importante respecto de la seguridad en el Mediterráneo es la repercusión de las crisis individuales en toda la región. El Mediterráneo es una gran caja de resonancia, debido sin duda a que en el sur existen una misma lengua, religión y cultura. El concepto de umma, comunidad de creyentes, vive en la calle de las ciudades árabes y se cohexionan ante cualquier ataque percibido contra la religión y la cultura de la hermandad islámica. El ejemplo más evidente es el conflicto árabe-israelí. Hay muchos aspectos de la seguridad en el Mediterráneo que no guarden relación con el conflicto árabe-israelí, pero este penetra todos los temas, negociaciones o conflictos en el resto del Mediterráneo, al igual que la crisis en Argelia influyó considerablemente en los demás países. En algunos casos estos efectos de desbordamiento trascienden la región del Mediterráneo. La agitación se transmite por medio de grupos de activistas islamistas argelinos en Francia o kurdos en Alemania. Ambos ejemplos explican la creciente percepción de merma de la seguridad personal en Europa, y que desde el 11-S también se ha trasladado a Estados Unidos.

\section{La impresión es que el empresario del norte se aprovecha de la debilidad del emigrante.}

Desde el sur se ve con preocupación el desarrollo de sentimientos xenófobos en los países de la Unión Europea. Se considera que sus compatriotas cada vez corren más riesgos de ser víctimas de ataques racistas. Además, con frecuencia aparece el sentimiento de ser explotado por el empresario del norte, que se aprovecha de la situación de debilidad del emigrante, aunque sin duda esta explotación no es la más dura que sufre el emigrante, sino la de las mafias organizadas que están encontrando que el tráfico de personas es un negocio más lucrativo que en el de las drogas. Como resultado de la migración, este fenómeno causa otra preocupación adicional en los países europeos, que ven el auge del racismo y el creciente apoyo a partidos de la ultraderecha como un peligro para sus instituciones y valores liberales. A su vez, esto puede aumentar el resentimiento frente a los inmigrantes (veáse Jerch et al. 2002, 2004).

Aunque los países europeos están habituados a recibir inmigrantes, al menos hasta ahora, la emigración islámica se resiste a su asimilación por razones culturales. Se trata de una inmigración con símbolos culturales externos que la diferencian (ropas, minaretes, etc.) cuya 
visibilidad inquieta a la población europea, que los percibe como un símbolo de «invasión de su espacio» cultural y de su vocación universalista. Lo que sucede es que existe una confrontación entre las dos únicas culturas en el mundo de vocación universalista: la occidental y la islámica. Ambas perciben la otra cultura como una amenaza a la suya propia. En el sur, la colonización fue percibida como una agresión cultural al Islam; hoy es en el norte donde, debido a la inmigración pacifica (a diferencia de la colonización), nace esa percepción de amenaza que es utilizada políticamente por la ultraderecha europea y que se manifiesta con violencia en la agresión al extranjero. Esta confrontación cultural no es nada nuevo, ya que tiene hondas raíces históricas, pero como siempre está basada en la ignorancia y en los desconocimientos mutuos. El diálogo cultural de la Declaración de Barcelona trataba de remediar este problema.

\section{La relación entre seguridad y desarrollo plantea un doble dilema sociopolítico y económico.}

En suma, se puede decir que todas las percepciones de amenaza tienen su origen en lo político y lo económico, ambos estrechamente vinculados. No cabe duda de que existe un importante vínculo entre desarrollo, tanto económico como político, y seguridad. Este vínculo explica de cierto modo la política mediterránea de la UE, que mediante el desarrollo económico de los PMNC busca crear una zona de estabilidad, seguridad y prosperidad en el Mediterráneo.

\section{La relación entre desarrollo y seguridad}

Según Robert McNamara (1968), seguridad es desarrollo y sin desarrollo no habrá seguridad. Se trata de una afirmación que actualmente ha ganado mucha popularidad y que es aceptada cada vez más, y eso a pesar de que según la nueva Estrategia de Seguridad de la Unión Europea, «la seguridad es una condición para el desarrollo». Algunos autores han identificado dos problemas vinculados con la relación entre seguridad y desarrollo en el contexto árabe: el dilema sociopolítico y el dilema relativo a los recursos económicos (Dessouki, 1993) ${ }^{6}$. El dilema sociopolítico se centra en los problemas de legitimidad y en la debilidad de las instituciones políticas en los países árabes ${ }^{7}$. La falta de legitimidad, que a su vez expresa el déficit de desarrollo político, se traduce en dos tipos de tensiones internas. La primera se relaciona con los conflictos a que

6 Dessouki distingue entre tres dilemas, es decir, añade el dilema militar, de escasa utilidad para el objetivo de este párrafo.

7 La legitimidad se refiere a las relaciones entre los que gobiernan y los gobernados, que se corresponde con el concepto árabe de al-urwa al-wathqa, es decir, en la medida en que la población se siente representada por la elite en el poder, aumenta el grado de legitimidad de esta. 
podrían dar lugar las nuevas tendencias de liberación política y la privatización económica. Es decir, la privatización económica y las reformas económicas liberales en general son fuente de conflictos, y la apertura política ofrece la oportunidad de expresarlos. Cuando se experimentan crisis económicas, por ejemplo, altas tasas de paro e inflación, aumenta también la inestabilidad política y el déficit de legitimidad. Un factor que determina este círculo causal es la forma en que se llevan a cabo estas reformas, expresado por Brumberg (1995) en el concepto de «estrategias de supervivencia». Es decir, las reformas se realizan para el fin de mantenerse en el poder y no para alcanzar el necesario cambio de las estructuras que necesita el sistema para funcionar bien. Se reforma para que todo quede igual.

Las reformas liberales significan la retirada del Estado del sistema económico: el mercado sustituye al Estado en la toma de decisiones. Este hecho pone en peligro el antiguo concepto de Estado benefactor (Lorca, 1995) y, en consecuencia, el clientelismo político, instrumento estabilizador utilizado por la élite gobernante para mantenerse en el poder. La introducción del mercado, aunque solo sea parcial, disminuye la corrupción, pero también aumenta los ingresos de la burguesía que no forma parte de la elite gobernante. El mercado libre da poder a una nueva clase social con poder económico pero no político.

La injusticia y de las desigualdades económicas dan lugar a otras tensiones, como la aparición de grupos islamistas militantes. La elite en el poder reacciona mediante la represión y la reducción de la apertura política. Egipto, Túnez y Argelia son ejemplos significativos al respecto. Por otro lado, las medidas que adopte el Estado frente a estos desafíos político-sociales aumentan el apoyo social a estas agrupaciones. Para hacer frente al declive de la legitimidad del régimen y disminuir el impacto de los movimientos islamistas a menudo se aplica otro mecanismo: se crea una amenaza externa, sea esta real o no, para distraer la atención de los problemas internos y fortalecer el sentimiento de integridad nacional, en el cual se basó durante mucho tiempo la legitimidad de la elite.

\section{Los recursos vitales de que disponen los países árabes son insuficientes para satisfacer las necesidades de la población.}

La correlación entre desarrollo y seguridad se explica por el dilema respecto de los recursos, que se relacionan con la situación económica y la transformación estructural. Los recursos vitales de los países árabes, como la tierra cultivable, el agua o los alimentos, son tan limitados que no pueden satisfacer las necesidades de la población. El crecimiento demográfico empeora aún más la situación. Todo ello conduce a inestabilidad política, migración y/o el creciente apoyo a los grupos políticos radicales. El impacto en la estabilidad política y, por lo tanto, en la seguridad, es manifiesto. Los dos dilemas, sociopolítico y económico, guardan estrecha relación. El mecanismo a través del cual se transmite son las denominadas «revueltas de la sémola». Los países árabes mediterrá- 
neos adolecen de una insuficiencia crónica de alimentos. La actuación del Estado benefactor se ha traducido en la puesta en práctica de una política de precios subsidiados para los alimentos y algunos servicios públicos, como los transportes. Esta política fue impuesta por la industrialización, que suponía la existencia de una creciente población urbana en crecimiento procedente de las zonas rurales, que desplazaba a hacia las ciudades a familias que sobrevirían con salarios bajos y que tenían escasa capacidad de compra.

\section{La industria nacionalizada vivía al amparo de los presupuestos del Estado.}

El gobierno necesitaba apoyar la industrialización y a la elite con salarios bajos y bienes intermedios baratos. Lo primero exigía una política de precios bajos para los alimentos, sostenidos por subsidios que permitían mantener los salarios en un nivel bajo. Lo segundo se basó en una industria nacionalizada ineficiente, que vivía al amparo de los presupuestos del Estado. Ambas políticas desembocaban indefectiblemente en déficit presupuestarios y de balanza de pagos difíciles de mantener a largo plazo, para cuyo financiamiento los países tuvieron que recurrir reiteradamente al Fondo Monetario Internacional (FMI). Este soluciona la falta de medios financieros de los Estados, pero impone los denominados ajustes estructurales, que implican disminuir los subsidios $\mathrm{y}$, en consecuencia, aumentar los precios de los alimentos y de los servicios públi- cos. De inmediato la población reacciona volcándose a las calles en las llamadas «revueltas de la sémola». Los Estados carecen de fuerzas de seguridad y antidisturbios bien entrenadas y eficaces, y solo cuenta con el ejército, que es utilizado para la represión interna. Las consecuencias inmediatas de todo esto son muertos y una espiral de violencia y represión. Con todo, desde 1990 y con la liberalización creciente de las economías y la estabilización de los precios, las revueltas han disminuido.

De lo anterior se deduce que para que haya seguridad es fundamental un orden político estable, basado en la legitimidad de los que gobiernan frente a los que son gobernados. Es decir, una relación en que el principal objetivo del régimen político es representar a la sociedad y en que el Estado sea capaz de movilizar los recursos para satisfacer las necesidades de la población. La relación entre desarrollo y seguridad, tal y como se ha analizado en el contexto árabe, se puede resumir en las palabras de Béchir Chourou (1998: 277): «el objetivo último de cualquier política es/ debe ser asegurar el bienestar del ser humano, y debemos considerar cualquier acción que impida o dificulte la consecución del bienestar humano como una amenaza a la seguridad».

Todo trabajo acerca de la seguridad en el Mediterráneo debe tener presente que la promoción de la cooperación y la seguridad debe basarse en la estabilidad y el desarrollo económico de la orilla sur y que solo una aproximación integrada de ambos problemas llevará a soluciones estables en dicha zona (Biad, 1996: 41). 
LAS PERCEPCIONES SOBRE LA SEGURIDAD EN LOS PAÍSES RIBEREÑOS

No resulta sencillo identificar los factores que influyen en la percepción de las amenazas y riesgos en el Mediterráneo. Para claridad de nuestro análisis dividiremos los países en dos bloques: norte y sur. Esto no quiere decir que ambos sean homogéneos. Por el contrario, la divergencia de intereses lleva a los países de cada bloque a mantener posiciones diversas frente a las distintas amenazas. Sin embargo, presentan rasgos comunes que trataremos de identificar.

\section{Se teme que la inmigración ilegal sea un medio de acceso del crimen organizado a las sociedades europeas.}

\section{Los factores de percepción en los países europeos}

Para todos los Estados europeos, la inmigración ilegal es uno de los principales problemas procedentes de la ribera sur del Mediterráneo. Fuera de la Europa mediterránea, Alemania, Finlandia, Holanda y Bélgica perciben con especial inquietud la supuesta amenaza de la inmigración ilegal. Inglaterra y Portugal, aunque menos afectados por los flujos migratorios procedentes de la ribera sur, también han expresado preocupación porque la inmigración ilegal sea un medio de acceso del crimen organizado a las sociedades euro- peas. Sin embargo, los datos de las encuestas relacionadas con el racismo y la xenofobia develan parte de los factores que conforman la percepción de amenaza en la UE.

En efecto, según encuestas realizadas a finales de los noventa por Eurobarómetro, un tercio de los ciudadanos de la UE se declaraban muy o bastante racistas, otro tercio un poco racistas y un último tercio, nada racistas. En general, las personas que se autodeclaraban racistas estaban insatisfechas con su situación personal y tenían temor a perder su empleo, aunque no se apreció ninguna correlación con el hecho de estar desempleado (de nuevo las percepciones: el temor a perder el empleo). La encuesta también revela que los jóvenes son menos racistas, al igual que las persona de mayor nivel de instrucción; sin embargo, no se aprecian diferencias significativas entre el medio urbano y el mundo rural. No obstante, las percepciones varían según el país europeo de que se trate.

Después de la Guerra Fría, Francia procuró modificar su definición de 'seguridad', de manera de dar mayor peso a los asuntos económicos y sociales. Ello supuso la confluencia de la inmigración sur -norte y de los intereses franceses por asegurar el suministro de energía del Oriente Medio y las líneas de comunicación marítimas. Esta política aumentó la atención hacia el Mediterráneo, no solo de Francia, sino de todos los países del bloque sur de la UE. A pesar de la preferencia por las iniciativas regionales, en Francia hay una elevada percepción de la importancia estratégica global del Mediterráneo, que 
deriva tanto de los intereses marítimos y costeros en el Mediterráneo como de sus intereses tierra adentro en la región: los observadores franceses a menudo ven la política hacia el norte de África como parte de un complejo más amplio de políticas hacia África, incluida la región subsahariana, donde Francia desea desempeñar un papel importante.

Los tipos de percepción y la política de seguridad franceses solo pueden entenderse considerando su autopercepción y su percepción de la historia. La política exterior francesa se caracteriza por la necesidad de jugar un papel de líder en el contexto internacional. Debido a su estatuto jurídico de miembro permanente del Consejo de Seguridad de las Naciones Unidas como potencia victoriosa en la Segunda Guerra Mundial, como potencia nuclear y militar europea y como lugar de nacimiento de la revolución burguesa, Francia quiere conservar una posición particular tanto en el plano internacional como a nivel europeo. Probablemente todo el enfrentamiento entre Francia y Estados Unidos respecto de la guerra de Irak en 2003 deba entenderse en este contexto. La autoimagen explica la importancia que tiene para el país galo mantener su statu quo en el escenario internacional.

\section{La política exterior francesa se caracteriza por la necesidad de ser líderes en el contexto internacional.}

El mantenimiento de este statu quo, así como la autoimagen de potencia líder, llevaron a percibir sucesos importantes en el escenario internacional como algo lento y estático y explican el intento de Francia por conservar el viejo orden mundial. Estos elementos son los dos pilares principales que determinan las decisiones de su política exterior. Se encuentran muchas pruebas de ello: por ejemplo, en los años cincuenta, en la época de los procesos de descolonización, Francia no reconoció el Zeitgeist a nivel mundial e intentó negarlo en la guerra de independencia de Argelia, que fue la guerra de independencia más brutal de la época. Este retraso en la percepción de sucesos importantes se manifestó también durante la reunificación alemana a finales de la década de los noventa. Por ello, Zbigniew Brzezinski (1998) afirma que los grandes perdedores del fin de la Guerra Fría fueron la Unión Soviética y Francia.

En la era de De Gaulle, el pensamiento francés sobre el Mediterráneo se caracterizaba por el deseo de deslegitimar el papel y la presencia de las superpotencias en la región, promoviendo la visión de la Méditerranée aux Méditerranéens (el Mediterráneo para los mediterráneos). En 1959 esta visión llevó a la retirada de la armada francesa del Mediterráneo para subrayar su decisión de no participar de ninguna manera en actividades militares integradas en la región. En consecuencia, la política gaullista favoreció el regionalismo antes que el globalismo, orientando el primero a construir relaciones duraderas con el Magreb y el Oriente Medio. El objetivo francés era separar las relaciones de seguridad en el Mediterráneo del planteamiento estratégico esteoeste, para que el Mediterráneo no se 
convirtiese en escenario de confrontación del conflicto entre las dos superpotencias.

Debido a su situación geoestratégica, la política de seguridad italiana se plantea de modo distinto a los demás países. Italia divide el Mediterráneo en sus partes oriental y occidental. Ello implica una fuerte integración en alianzas, lo cual, por su parte, juega un papel importante en la percepción de amenaza. La política de seguridad del país se determina por un alto grado de lealtad hacia sus responsabilidades en el contexto internacional (Greco y Guazzone, 1992:69). Este fenómeno se basa esencialmente en que los líderes italianos son conscientes de que, debido a su situación geoestratégica y a su posición de poder limitado en el ámbito internacional, solo pueden asegurar la seguridad nacional y los intereses del país mediante una cooperación estable con occidente. La discontinuidad en la participación en alianzas que se observa desde los años setenta, y que podría explicarse por el frecuente cambio de líderes, se caracteriza por la oscilación entre las opciones europeas, por un lado, y las opciones estadounidenses, por el otro. Köhler (1988; 1993) resume dicho cambio en tres fases: de 1973 a 1980, Italia se orientó más hacia Europa, no solo por su interés creciente en el espacio mediterráneo dentro de la CEE (que creó la Política Mediterránea $\mathrm{Global}^{8}$ ), sino por su interés en una política de estabilidad como la promovida por la Cooperación Política Europea. El aumento del interés italiano en el Mediterráneo marchó a la par del conflicto en el Oriente Medio (Guerra de YomKippur y crisis del petróleo).

\section{El endurecimiento de la Guerra Fría dio lugar a una nueva fase de la política de seguridad italiana.}

A comienzos de los años ochenta, con el nuevo endurecimiento de la Guerra Fría a causa de la invasión soviética en Afganistán y el doble acuerdo de la OTAN, se puede hablar de una nueva fase de la política de seguridad italiana, en la que nuevamente se buscó el acercamiento a la política de seguridad regional de los Estados Unidos. Con este nuevo cambio Italia esperaba aumentar su importancia en el contexto regional; además, Italia en el debate interno de la OTAN sobre misiones out-of-area, no vislumbraba una posibilidad de mejorar a largo plazo su posición en Europa. Esta fase se extendió hasta 1986. En 1985 se dieron ya las primeras señales que indicaban este nuevo cambio, que se inició con el delicado asunto del Achille-Lauro, en el que se enfrentaron directamente tropas estadounidenses y la policía italiana.

8 En la cumbre del Consejo Europeo de París, de 1972, se decidió que la política mediterránea debería basarse en un planteamiento global y equilibrado. Así nace la «Política Global Mediterránea» (PGM), que consistía en la apertura de la CEE a los productos industriales y el acceso preferencial de productos agrícolas, ayuda financiera y cooperación económica. Se notaba el importante cambio que introdujo la PGM a causa de la insuficiencia e incapacidad de los antiguos acuerdos bilaterales para abarcar todo el abanico de intereses de la CEE en la región del Mediterráneo. La cumbre de París inició la segunda fase de la política mediterránea, que duraría hasta 1978. 
El principal factor de percepción italiana fue el riesgo de perder la conexión con los socios europeos. A este fenómeno se puede llamar el «temor a la marginación» frente a los países centro y noreuropeos ${ }^{9}$. Este temor no es un fenómeno nuevo: ya en la fase de distensión de los años setenta los líderes italianos intentaron aproximar militarmente más la seguridad europea con la del Mediterráneo (Köhler, 1993: 122). Por medio de una declaración en que se reevaluaba la amenaza soviética en el Mediterráneo, Italia crearía la misma distinción de categorías entre el frente central y el flanco sur dentro de la OTAN. La caída del Muro de Berlín, la reunificación alemana y la creciente tendencia de Alemania a mirar hacia el este, provocaron en Italia una nueva percepción de marginación. Para crear un polo opuesto al de la Alemania unificada, Italia buscó desempeñar un papel autónomo en la cooperación con los países de Europa del este. Por tal motivo, apoyó la idea de un hexágono (Italia, Austria, Yugoslavia, Hungría, República Checa y Polonia).

Aparte de los factores mencionados, la política de seguridad interna también desempeña un papel importante en la comprensión de las percepciones italianas. Desde el fin de la Segunda Guerra Mundial el desarrollo de la política interna se ha caracterizado por el desacuerdo entre el gobierno y la oposición en decisiones importantes. En suma, tanto las angustias de la marginación en el contexto euroAtlántico como las políticas de seguridad interna son factores determinantes de la percepción italiana de amenazas y riesgos.

\section{El consenso de que «España ha vuelto a Europa» expresa su nuevo activismo en el escenario internacional.}

En los últimos años, España ha dado muestras de un nuevo activismo en el escenario internacional. Su dinamismo se expresa en el consenso de que 'España ha vuelto a Europa' después de siglos de aislamiento respecto de los temas europeos, lema que también se podría utilizar para describir "la reorientación" del gobierno español tras el cambio de gobierno en marzo 2004. La Guerra de Golfo, a principios de los años noventa, dio a España un papel más activo en asuntos de seguridad, tanto interior como exterior. Tras la experiencia de dicha guerra, el entonces Ministro de Defensa, Julian García Vargas, declaró que España tenía que ampliar su filosofía de seguridad; que ella no se puede limitar a un espacio territorial nacional (El País, 25-4-1991). En ciertos círculos, principalmente militares y conservadores, la amenaza soviética de la Guerra Fría fue sustituida por la noción de una 'amenaza del sur', referida al Magreb. Los dos enclaves españoles en el continente africano, Ceuta y Melilla,

9 Véase Aliboni (1992b: 5). Este autor observó una marginalización en el contexto euro-atlántico y una singularización respecto de la amenaza del sur por los Estados europeos ya antes de las transformaciones globales de los últimos años. 
junto con la proximidad de Canarias a la costa africana, tienen en esta percepción un objetivo concreto. Aparte de una posible crisis en estos enclaves, los estrategas españoles no pueden ofrecer ningún otro escenario concreto.

\section{En los últimos diez años España se ha convertido en zona receptora de inmigración.}

Tras la transición a la democracia en 1976, la política exterior española no ha contribuido a mejorar la negativa percepción acerca de los vecinos del sur, asociada a problemas territoriales y al subdesarrollo. Por el contrario, las ambigüedades de esta política han contribuido a que la percepción española del «moro» y de la frontera sur continúe alimentándose de noticias negativas basadas en sucesos aislados. Adicionalmente, España, que tradicionalmente ha sido exportadora de mano de obra, en los últimos diez años se ha convertido en una zona receptora de inmigración. El crecimiento económico español y su integración en la CEE han contribuido a que un buen número de magrebíes se instalen en España, nueva frontera sur comunitaria, en muchos casos en forma ilegal. Y aunque el número de inmigrantes es inferior al que llega al resto de Europa, los prejuicios de los españoles hacia el «moro» se convierten en comportamientos xenófobos y racistas.

Rafael Grasa (1992) y Carlos A. Zaldívar (1991) señalan que en la percepción española aún están presentes elementos de la política exterior y de seguridad del régimen franquista y de la época de la transición, que están influyendo en las actuales decisiones de política exterior. La percepción de enfrentamiento con los Estados Unidos que se percibe en la izquierda española se explica, primero, por la política estadounidense en los países de América Latina, que tiene fuerte influencia en la opinión pública española y, en segundo lugar, porque a diferencia de otros países europeos, España no tiene «deudas» pendientes con Estados Unidos relacionados con la liberación de los poderes del eje durante la Segunda Guerra Mundial o con el apoyo económico del plan Marshall (Zaldívar, 1991:212).

La percepción de amenaza de la Unión Soviética, ni siquiera durante el liderazgo de Breshnev, se percibió como la amenaza principal, y desapareció en los tiempos de la «glasnost» y la «perestroika», lo que se explica por la distancia geográfica y la ausencia de conflictos históricos. La presencia de la ex Unión Soviética en el Mediterráneo tampoco se percibió como amenaza debido a la distancia y a que en el Mediterráneo occidental no había bases soviéticas. Además, la dictadura de Franco entre 1939 y 1975, con el fuerte aislamiento internacional de España, sobre todo respecto de Europa, impidió que en el país surgiera una «cultura» de Guerra Fría (Zaldívar, 1991: 212). Estas circunstancias son decisivas para la actual «definición de la situación mediterránea» por parte de España. Muestran también que en España el «vacío de amenaza», surgido al desaparecer el Pacto de Varsovia de la mente de los estrategas occidentales, solo se sintió marginalmente. La 
amenaza aquí se percibe de manera diferente. Actualmente, en el país hay conflictos locales-nacionales por un lado y por otro conflictos globales, es decir, al margen de las responsabilidades españolas en la OTAN y la UE.

\section{Los gobernantes debieron compensar su frágil legitimidad mediante un consenso nacional.}

\section{Los factores de percepción de los PMNC}

El factor más determinante se basa en que el concepto de seguridad de los países en desarrollo se diferencia mucho del que se ha creado en occidente después de la Segunda Guerra Mundial. Según Mohammed Ayoob (1993) esta diferencia se manifiesta en los siguientes rasgos: 1) comparados con los líderes de los Estados industrializados, los líderes de los países en desarrollo perciben más una amenaza proveniente de fuerzas internas; y 2) la fuerte tendencia hacia conflictos intra-estatales con efectos de desbordamiento en el ámbito regional de los Estados en vías de desarrollo.

Ayoob afirma que el origen de los conflictos intra-estatales es la herencia colonial, en particular el proceso de transmisión de poder realizado por la metrópoli al primer gobierno independiente. Un factor adicional estriba en la necesidad de los gobernantes de compensar su frágil legitimidad mediante un consenso nacional, que en el pasado a menudo se consiguió gracias a problemas externos. Un ejemplo de dicho fenómeno es la posición de Marruecos, que renueva de vez en cuando sus reivindicaciones sobre Melilla y Ceuta y trata de aglutinar a la población recurriendo al conflicto del Sahara occidental. La legitimidad política se relaciona de manera inseparable con el grado de legitimidad del sistema político. Y esto es, según Ayoob, un producto fundamental del proceso de «construcción del Estado-nación» (1993: 33), que muchos países en desarrollo aún no ha finalizado. Es decir, las percepciones de los PMNC en materia de seguridad se refieren básicamente a tres aspectos: la dimensión externa subregional (sur-sur), la dimensión externa norte-sur y las amenazas internas. Nos detendremos en cada uno de ellos, empezando por las primeras.

Las tensiones entre los PMNC tienen diversas fuentes. En primer lugar, una herencia colonial de fronteras disputadas transmitida a los actuales Estados, que siguen reivindicando reajustes de demarcación. Por otro lado, lejos de conformar un bloque homogéneo en materia de política exterior, el mundo árabe está muy fragmentado, padece un déficit democrático importante y las potencias regionales se disputan el equilibrio de poder, tratando de alcanzar una posición hegemónica a nivel subregional (Lorca y Escribano, 1998a y 1998b), al punto que dicha rivalidad entre Estados se ha comparado con la rivalidad franco-alemana o argentino-brasileña, que la integración regional en el seno de la UE y el Mercosur han conseguido mitigar (Vasconcelos, 1999). No obstante, las per- 
cepciones de amenaza regional difieren entre las partes oriental y occidental de la ribera sur.

En el Mediterráneo suroriental y el Levante, la cercanía del conflicto árabeisraelí polariza en mayor medida la actitud de los gobiernos árabes que en el Magreb. Pese a las agresiones intra-árabes (Siria-Líbano) y a las tensiones intramusulmanas (Siria-Turquía o EgiptoSudán), la percepción de amenaza externa más creíble procede de Israel, la potencia militar regional. Sin embargo, como lo han demostrado los acontecimientos del 11 de septiembre, el conflicto árabe-israelí tiene claros efectos de desbordamiento, al difundir entre la población árabe y musulmana la imagen de que occidente no solo trata a Israel y los países árabes con un doble rasero, sino que Estados Unidos apoya diplomática y militarmente a aquel. En consecuencia, la amenaza procedente de Israel se amplía de forma difusa a los Estados Unidos y, por extensión, al conjunto del mundo occidental. Aunque hay problemas fronterizos, en general relacionados con el acceso a recursos escasos como el agua, estos quedan velados por la preeminencia de la amenaza israelí.

En el Magreb, las cosas son algo distintas. Autores como A. Benramdane (1992) o M. Elmandjra (1989) confirman que en Marruecos las cuestiones de seguridad se centran en asuntos relacionados con la integridad territorial. Concretamente en la cuestión del Sahara occidental, debido al apoyo del régimen argelino a los saharauis, y en las fronteras con Argelia. Los autores también se hacen eco de los problemas de cooperación con la
UE, pero en menor medida. La percepción de amenaza está determinada por la amenaza sobre sus fronteras, que procede del vecino del sur. En Marruecos, el debate nacional permite distinguir claramente dos posiciones; por un lado, su posición de país no-alineado desde finales de los años cincuenta y, por el otro, un acercamiento paulatino hacia occidente. El caso de Túnez se asemeja al de Marruecos. La integridad territorial (y sus fronteras terrestres y marítimas, sobre todo con Libia) ocupa el primer lugar de la «lista» de preocupaciones de seguridad nacional, aunque la relación con occidente también es tema de debate. Por su parte, las preocupaciones argelinas se han centrado en la búsqueda de una posición (tanto política como económica) en la esfera internacional, como lo reflejan por ejemplo sus esfuerzos en el movimiento de los países no alineados. Los investigadores argelinos prácticamente no han tratado temas de seguridad a nivel regional, pese a que Marruecos y Argelia se disputan la hegemonía en el Magreb.

\section{En Marruecos pueden distinguirse su posición como país no alineado y su acercamiento paulatino a Occidente.}

A nivel global, en el debate estratégico de los investigadores árabes se ve la oposición entre el concepto de seguridad nacional propio de cada Estado, el al amn al watani, y la seguridad del conjunto de los países árabes, el al amn al qawmi. Mientras que el primero concibe a cada Estado como una entidad distinta y autó- 
noma dentro de sus fronteras, el segundo se refiere a la seguridad árabe global. Este concepto global contiene aspectos de seguridad contradictorios, no pudiéndose ignorar que las políticas de los Estados árabes se determinan por las preocupaciones e intereses propios de cada uno de ellos.

\section{Cada Estado árabe tiene su enemigo árabe además del enemigo común israelí.}

La definición árabe de seguridad nacional se basa, entre otras cosas, en la existencia de una lengua común y las mismas creencias y sistema de valores. $\mathrm{Cu}$ riosamente, para apoyar su perspectiva nacional, los investigadores árabes introdujeron la idea de que actualmente el mundo se compone de grandes entidades. Fuera de estos grupos no queda lugar para pequeños Estados aislados. Pero es evidente que el concepto árabe de seguridad nacional tiene el mismo y escaso contenido que los conceptos que estaban en la línea del nacionalismo árabe, con sus corrientes nasseristas o baathistas. Así, en el ámbito institucional del mundo árabe, se puede observar que el término «seguridad» no aparece ninguna vez en el pacto de la Liga Árabe. Al interior de la Liga Árabe las percepciones de amenaza varían de país a país. Según la intensidad de la coyuntura, cada Estado árabe tiene su enemigo árabe, además del enemigo común israelí. Este fenómeno se aprecia claramente en el Magreb, por ejemplo, en las relaciones entre Marruecos y Argelia o entre Túnez y Libia. Para terminar con las tensiones y establecer una «zone de paix et securité» (Khader, 1993: 56) en el Magreb, los países magrebíes ratificaron en 1989 el acta constituyente de la Union du Maghreb Árabe (UMA). Además de una intensa cooperación económica, técnica y cultural, el acta también contempla aspectos de política de seguridad.

Pero aparte de estos conflictos entre Estados árabes, a nivel nacional hay que enfrentar la amenaza interna, que en términos generales puede definirse como una amenaza al sistema político nacional y, más concretamente, como problemas planteados por el islamismo político. A este nivel, los trabajos estratégicos vinculan «seguridad» con «desarrollo» y concluyen que la economía constituye un principio fundamental de la seguridad de los Estados árabes; es decir, que el mayor riesgo para su seguridad es la dependencia económica. El endeudamiento también se considera una forma de dependencia y, por tanto, como una amenaza, puesto que un país con deudas no es un país libre. Al lado de estas consideraciones económicas hay una dimensión política que abarca aspectos como la ausencia de integración nacional (construcción nacional), la falta de un consenso nacional, la existencia de un grado de politización muy débil, la insuficiente legitimación del aparato del Estado o la herencia de conflictos no resueltos.

Esta aproximación al debate sobre seguridad y defensa en el contexto árabe nos muestra que, a diferencia de lo que ocurre en los países ribereños de la cuenca norte las percepciones de ame- 
naza tienen un elemento interno importante.

\section{Una amenaza al funcionamiento de las instituciones es una amenaza para la seguridad nacional.}

Un aspecto que tienen en común las constituciones de los PMNC es que dan al jefe del Estado, bien al presidente, bien al rey, el papel de comandante de las fuerzas armadas, de defensor del Estado, la independencia, la integridad territorial y la soberanía. Según los textos fundamentales, cada ciudadano tiene la obligación de defender la nación y la integridad territorial del Estado. Y en cada texto constitucional la noción de seguridad nacional se relaciona con el mantenimiento de las instituciones estatales, puesto que una amenaza al funcionamiento de las instituciones representa una amenaza para la seguridad nacional. Ante tal situación, el jefe de Estado puede tomar medidas excepcionales, como si se tratase de una amenaza a la integridad territorial. El hecho de que las constituciones den importancia excesiva a las instituciones estatales subraya el carácter de "amenaza interna" de la seguridad nacional en los países árabes del Mediterráneo. Para la mentalidad árabe, no sólo son cuestión de seguridad las amenazas a las fronteras, sino también el propio concepto de Estado, su statu quo y las amenazas a estas estructuras son fuentes de amenaza.

Respecto de la función de las Fuerzas Armadas, se comprueba que ellas ocupan un lugar muy importante en la vida política de los Estados árabes, en parte debido a un reciente pasado colonial y a la lucha por la independencia. El ejército controla importantes resortes de poder político y económico, como se aprecia en el hecho de que numerosos dirigentes árabes proceden del estamento militar.

En el mundo árabe, la seguridad y la estabilidad del régimen político están estrechamente relacionadas. Esta relación se ha convertido en el instrumento para mantener el statu quo, donde la alternancia y la pluralidad política se consideran una amenaza a la unidad nacional y a la estabilidad interna. Esta relación seguridad - estabilidad ha adquirido importancia en los últimos años debido al crecimiento de los movimientos islámicos, a las presiones de democratización de los regímenes políticos y a los problemas económicos y demográficos, a los cuales los países no encuentran una solución a corto plazo. Esta inestabilidad es percibida por el poder árabe como "amenaza interna" al statu quo y a su concepto de Estado.

Aunque el problema de seguridad en la ribera sur del Mediterráneo es principalmente un problema sur-sur, desde el fin de la Guerra Fría y sobre todo durante la guerra del Golfo, de 1991, aumentó la percepción de amenaza proveniente del «norte». En el mundo árabe existe la impresión de que en el contexto europeo de seguridad se busca desenfrenadamente un enemigo en el ámbito árabe-islámico. Sin duda, el comportamiento de los europeos y sus aliados se considera como un complot occidental contra los árabes (Saaf, 1995: 24).

Una percepción adicional de amenaza a nivel vertical se relaciona con el bien- 
estar de los árabes que viven en Europa. El aumento de las tendencias racistas y xenófobas en toda Europa en los últimos años, sobre todo el éxito de Le Pen y el Front National y su mensaje contra los norafricanos que viven en Francia, en la ribera sur provocan grave preocupación. El aumento de la xenofobia, las acciones contra los beurs (árabes) y los inmigrantes y las políticas contra la inmigración subrayan esa percepción de la división del Mediterráneo.

\section{En los países de la orilla sur existe la impresión de que Occidente lleva a cabo una guerra sin declarar contra el mundo árabe.}

Este fenómeno crea en los países de la orilla sur la idea de una guerra sin declarar que occidente está llevando a cabo contra el mundo árabe, hecho que se ve nutrido por las acciones terroristas en las sociedades occidentales, que a menudo no saben distinguir entre Islam y terrorismo. Además, en la mente de los árabes surge la impresión de que son rechazados por los europeos. Por un lado, los medios de comunicación crean aspiraciones de una vida mejor en el norte y, por el otro, ese mismo norte les deniega la realización de sus aspiraciones. Este rechazo y la destrucción de sus esperanzas y de sus sueños de una vida mejor crean unl sentimiento de agresión unilateral que afecta a la vida cotidiana. La amenaza es, por tanto, resultado de esta dicotomía básica y de los conflictos que ella provoca. Comparativamente, la amenaza militar es menos importante. El riesgo surge del desequilibrio de la balanza de poder y de cuestiones de hegemonía, reforzadas en la imaginación popular por una fuerte, aunque reciente, conciencia histórica.

\section{A MODO DE CONCLUSIÓN: LA DICOTOMÍA «NOSOTROS»Y «ELLOS»}

Nuestro análisis confirma la hipótesis de que entre los países ribereños del Mediterráneo existe una separación respecto de las shared images (imágenes compartidas) de percepciones y distancias. Las percepciones de amenaza del norte se pueden resumir en la «inmigración» y el «extremismo islámico», con sus derivaciones hacia el terrorismo. En los países ribereños del norte estas percepciones provocan un aumento de sus mecanismos de protección para intentar reducirlas, lo que a su vez genera un incremento de las amenazas percibidas por los países de la orilla sur, creándose así un dilema de seguridad. Por ejemplo, el intento de controlar la inmigración ocasiona un problema de inmigración ilegal y repercute negativamente en la situación económica de los países del sur, con lo cual se agrava la inestabilidad política y la UE debe recurrir a nuevas medidas de protección, que a su vez son percibidas como una amenaza por los países afectados.

Quizás con la única excepción del terrorismo internacional, las percepciones de amenaza no resultan muy plausibles, como se ha visto recientemente. Existen diferencias entre la percepción y la realidad, resultado de los diversos factores expues- 
tos. ¿Cómo se puede explicar está situación? Como vimos al analizar los países ribereños, la cuenca del Mediterráneo atraviesa por un proceso de marginación relativa que retrasa su progreso, tanto político como económico. ¿Qué provoca esta marginación? ¿La apertura de Europa hacia el este ¿afecta al Mediterráneo?; y si es así, ¿cómo? En el pasado se argumentaba que el este era políticamente parte de Europa y, por tanto, que pertenece a la Unión Europea, una entidad que llamaremos «nosotros». No solo eso, sino que es una región que parte de Europa percibe como «el hijo pródigo que vuelve a casa»: regresa a la ideología occidental tras su experiencia con el marxismo. Existe la firme voluntad política de apoyar este regreso, porque de esta manera la Unión Europea se apoya a sí misma, pues el este es, ante todo, «nosotros». Este razonamiento es difícil de aceptar para algunos países mediterráneos de la UE, que no perciben tan fácilmente el Este como «nosotros», e incluso pueden llegar a percibirlo como «ellos». Sin embargo, diríamos que este punto de vista es el de una minoría.

\section{A algunos países mediterráneos de la UE les cuesta percibir a los países del este como «nosotros».}

De cualquier modo, evidentemente existe una dicotomía de intereses dentro de la UE: por un lado los países que se dirigen hacia Europa del este y, por otro, los países cuyos intereses se encuentran en la frontera sur. El "bloque mediterráneo", formado por Francia, Italia, España y Portugal, tiene un gran interés por la zona del Mediterráneo occidental, y Grecia lo tiene en la parte oriental. El «bloque central» es encabezado por Alemania, que es el país que tiene mayores intereses en el este, acompañado por el Reino Unido, Dinamarca, Bélgica y los países escandinavos. Estas diferencias no se refieren exclusivamente a los intereses, sino más bien a las preferencias. Pero ¿hasta qué punto pueden separarnos estas diferencias de prioridades?

Cuando se analiza la ayuda comunitaria, no parece que la UE haya sido demasiado generosa con el Mediterráneo, pero si ampliamos el espectro de medidas, las diferencias son todavía más sorprendentes. Es difícil pensar que el Mediterráneo podría recibir de la UE o de occidente volúmenes de ayuda tan importantes como los previstos para el este, pero más difícil aún es considerar para el Mediterráneo sur un estatus semejante al que su ampliación prevé para los países candidatos de Europa oriental. Las circunstancias son muy diferentes y también lo es el juego de intereses económicos, políticos e ideológicos. Occidente nunca destinará grandes sumas de dinero a zonas de tan alto riesgo como los países de la cuenca sur del Mediterráneo. Las percepciones de occidente expuestas en capítulos anteriores (inestabilidad interna, fundamentalismo islámico y economías que no son capaces de consolidar un crecimiento y ajustarse a la presión demográfica) son negativas y, por tanto, no se da una situación favorable para captar capitales extranjeros.

Además, la asistencia para el desa- 
rrollo es totalmente insuficiente para alcanzar el desarrollo económico de un país. Hoy día, se necesitan capital y tecnología, y no debe olvidarse que ambos están en manos de actores privados. La financiación pública solo se puede considerar como complementaria y está principalmente dirigida a mejorar los servicios públicos y la formación de capital humano: la ayuda pública abarca el déficit que deja el capital privado. Por ello, no cabe duda que es necesario atraer capitales, y en ello radica la gran diferencia entre «nosotros» $\mathrm{y}$ «ellos» (Lorca, et al. 1996). Esta diferencia en la capacidad de atraer capital privado tiene sus raíces en la separación cultural y religiosa: el fundamentalismo musulmán es percibido como peligroso y subraya las diferencias con «nosotros», despertando en occidente la conciencia del concepto «ellos».

Otro freno a la integración económica fronteriza es el concepto árabe de Estado. En la cultura árabe el Estado es tradicionalmente intervencionista, lo que dificulta la integración económica transfronteriza. Unida a las ideas nasseristas, esta concepción intervencionista del Estado, dio lugar al denominado socialismo árabe, que dio protagonismo casi único al Estado como promotor del desarrollo económico y ha llevado al mundo árabe a enormes despilfarros de recursos y a la creación de una burocracia ineficaz, en algunos casos corrupta, que actúa con dureza y no deja funcionar eficazmente al mercado. El concepto de un Estado paternalista, distribuidor de beneficios y prebendas, cuyo objetivo es asegurar las redes del clientelismo político, y al que se acude constantemente para la solución de cualquier tipo de problema, lleva a consecuencias sociales perversas. La burocracia se convierte en intermediaria entre el ciudadano y el presupuesto del Estado como distribuidora de privilegios, con la consiguiente aparición de la corrupción. Además, esta estructura refuerza los lazos familiares, de clanes y tribales, lo que dificulta la modernización del aparato del Estado.

\section{En la cultura árabe el Estado es tradicionalmente intervencionista.}

En el mundo árabe los regímenes poscoloniales han fracasado en sus objetivos de desarrollo económico y solución de los problemas materiales de sus ciudadanos. La presión demográfica excesiva, el déficit alimentario provocado por una política industrial radical y la convicción de que la agricultura no tiene cabida en la industrialización han contribuido al fracaso de los gobiernos. Lógicamente, la población, en particular los jóvenes con buen nivel de instrucción, ha reaccionado rechazando todo lo que representan estos regímenes: corrupción, formas de gobierno occidentales, etc. La solución se busca en las propias raíces islámicas y se acompaña de un rechazo de la Westernization de la sociedad árabe. Todo ello dificulta la creación de economías transfronterizas. La Unión Europea, en una posición defensiva y muy escéptica, no se decide a ir al Mediterráneo con su tecnología y su capital, elementos esenciales para el desarrollo de esta periferia. 
El problema obedece a la ausencia de un planteamiento adecuado respecto de la forma de atraer capital privado. La percepción de un alto riesgo-país en la zona paraliza las decisiones de inversión de las empresas occidentales y por ello se torna cada vez más necesario establecer un diálogo entre los pueblos del Mediterráneo. La UE debe procurar evitar la marginación de su frontera sur y esforzarse por integrarla económicamente. El aislamiento del mundo árabe mediterráneo se incrementará a raíz de los sucesos del 11 de septiembre. Las empresas occidentales percibirán en el mundo árabe amenazas para sus intereses y sus ejecutivos. Algunas de ellas incluso han prohibido a sus empleados viajar a determinados países islámicos.

Sin duda alguna, es preciso encontrar un camino para llegar a entender mejor los valores de los vecinos. El acercamiento debe empezar por aumentar los conocimientos acerca de «ellos» y por profundizar el diálogo. A nuestro juicio, la Conferencia euro-mediterránea de Barcelona es el comienzo de un largo y difícil, pero necesario, camino de acercamiento entre ambas riberas.

Hemos visto cómo desde el término de la Guerra Fría se introdujeron nuevos conceptos en las discusiones académicas sobre seguridad, posibles escenarios de conflictos y percepción de amenazas. Los modelos explicativos de la «nueva» situación son muy variados y se han producido cambios fundamentales en los planteamientos de seguridad. Durante la Guerra Fría, la situación de seguridad consistía en una amenaza militar a la integridad terri- torial procedente de un enemigo ideológica y geográficamente bien definido. En cambio, actualmente parece muy difícil que los políticos e investigadores puedan definir terminológica y geográficamente la nueva situación. En el ámbito de la política de seguridad, se habla de amenaza, riesgos (en plural), peligro o desafío, pero difícilmente se pueden definir y diferenciar estos términos de contenido más que difuso.

\section{Hay que encontrar un camino para entender mejor los valores de los vecinos del sur.}

Lo mismo ocurre respecto del «objeto» amenazado. ¿Es la integridad territorial o, como dice Wæver et.al., la identidad de las sociedades la que está amenazada? ¿La cultura? ¿A través de quién y de qué? ¿Mediante la «infiltración» de inmigrantes de culturas extrañas? Mediante mecanismos de integración transnacionales como la UE que, según Wæver et al. (1993), conducen a un renacimiento del elemento «nacional», como se vio en el referéndum de Maastricht en Dinamarca, o en el rechazo noruego a entrar en la UE? ¿Qué es identidad? ¿Significa identificarse con una civilización apoyar a la idea del choque de civilizaciones de Huntington (1996)?

La tesis del choque de civilizaciones defendida por Huntington no tiene en cuenta las causas del aumento de las tendencias islamistas en el Mediterráneo, señaladas en capítulos anteriores. A nuestro juicio, los problemas de seguridad en el 
Mediterráneo no consisten en una lucha entre la civilización occidental y el Islam, sino que forman parte del proceso de modernización de las sociedades de la ribera sur y, como nos muestra la historia europea, estos procesos pueden ser muy conflictivos. En el Mediterráneo no existe confrontación entre occidente e Islam ni hay unas fronteras sangrientas del Islam (the bloody frontiers of Islam) (Huntington, 1996), sino una confrontación norte-sur que se manifiesta por diferentes conflictos, incluido el cultural.

\section{REFERENCIAS BIBLIOGRÁFICAS}

Aliboni, R. (ed.) (1992a), Southern European Security in the 1990s, Londres, Pinter.

Aliboni, R. (1992b), Southern European Security. Perceptions and Problems, en Aliboni, R. (1992a).

Aliboni, R., Joffé, G. y Niblock, T. (eds.) (1996), Security Challenges in the Mediterranean Region, Londres, Frank Cass.

Ayoob, M. (1993), Unravelling the concept: 'National Security' in the Third World, en Korany et al. (eds.) (1993).

Benramdane, A. (1992), Le Sahara Occidental: enjeu maghrébin, Paris, Karthala.

Biad, A. (1998), L’Algérie et la Méditerranée: Perception des vulnérabilités et besoin de coopération, en Marquina, A. (ed.) (1998).

Biad, A. (1996), Security and Co-operation in the Mediterranean: A Southern Viewpoint, en Aliboni, R. et al. (eds.) (1996).

Blunden, M. (1994), Insecurity on Europe's Southern Flank, en Survival, Vol. 36, $\mathrm{N}^{\circ} 2$.

Brumberg, D. (1995), Authoritarian Legacies and Reform Strategies in the Arab World, en Brynen, et al. (eds.) (1995).

Brynen, R., Korany, B., Noble, P. (eds.) (1995), Political Liberalization and Democratization in the Arab World, Volumen 1, Theoretical Perspectives, Londres, Boulder.
Brzezinski, Z. (1998), El gran tablero mundial. La supremacía estadounidense y sus imperativos geoestratégicos, Barcelona, Paidos.

Chourou, B. (1998), Redefining Mediterranean Security: A View From the South, en Marquina, A. (ed.) (1998).

Cosidó, I. (1995), La amenaza del Sur, en Política Exterior, Vol. 45, $\mathrm{N}^{\circ}$ 9, junio/julio.

Daase, C., Feske, S., Moltmann, B. y Schmid, C. (eds.) (1993), Regionalisierung der Sicherheitspolitik. Tendenzen in den internationalen Beziehungen nach dem Ost-West-Konflikt, Baden-Baden, Nomos.

Dessouki, A. (1993), Dilemmas of Security and Development in the Arab World: Aspects of the Linkage, en Korany, B. et al. (eds) (1993).

Elmandjra, M. (1989), Retrospective des Futurs, Casablanca, Ed. Ouyoun.

Escribano, G. (coord.) (1999), El Área de Libre Comercio Euromediterránea, Madrid, Centro Español de Relaciones Internacionales (CERI).

Farrar-Hockley, D. (1994), Future Instability in the Mediterranean Basin, en European Security, Vol. 3, No. 1, Spring.

Fisas, V. (1991), El mito de la amenaza del sur. Rearme y comercio de armamentos en el Mediterráneo Occidental, Zaragoza, Seminario de Investigación para la Paz.

Gantzel, K. (1972), System und Akteur: Beiträge zur vergleichenden Kriegsursachenforschung Düsseldorf, Bertelsmann.

Grasa, R. (1992), La perception de la menance en Espangne, en ARÈS (Grenoble), Vol. XIII, N ${ }^{\circ}$ 3 , diciembre.

Greco, E. y Guazzone, L. (1992), Continuity and Change in Italy's security policy, en Aliboni, R. et al. (eds.) (1992a).

Huntington, S. (1996), The Clash of Civilizations and the Remaking of World Order, Nueva York, Touchstone.

Jerch, M., Escribano, G. y Lorca, A. (2002), The Impact of Migration from the Mediterranean on European Security, en Vasconcelos, A. (coord.) (2002).

Jerch, M. y Lorca, A. (2004), A North-South Migration Dilemma, en: bitterlemonsinternational.org, marzo 19, Ed. 11 Vol. 2.

$<w w w . b i t t e r l e m o n s-i n t e r n a t i o n a l . o r g /$ inside.php?id=130) > (consultado 22 de marzo 2004). 
Jervis, R. (1976), Perception and Misperception in International Politics, Princeton, Nueva York, Princeton Uiniversity Press.

Kaufmann, F. (1973), Sicherheit als soziologische und sozialpolitisches Problem. Untersuch-ung zu einer Wertidee hochdifferenzierter Gesellschaften, Stuttgart, Enke.

Khalilzad, Z. y Lesser, I. (eds.) (1998), Source of Conflict in the $21^{\text {st }}$ Century. Regional Futures and U.S. Strategy, Santa Monica, Cal., RAND.

Khader, B. (1994), L'Europe et la Mediterranée. Géopolitique de la proximité, París, l'Harmattan.

Khader, B. (1993): L'Europe face aux nouveaux risques: Le defi du Maghreb, Bruselas, Centre d'etudes de Défense, Veiligheid en Stratégie, Sécurité ez Stratégie, $\mathrm{N}^{\circ} 1$.

Köhler, M. (1988), Italienische Sicherheitspolitik in den 80er Jahren. Aktivitäten, Perzeptionen, Positionen, Berlin, Berghofstiftung (Arbeitspapier No 32).

Köhler, M. (1993), Sicherheitszone Mittelmeer Italiens mediterrane Sicherheitspolitik, en Daase et al. (eds.) 1993.

Korany, B., Noble, P. y Brynen, R. (eds.) (1993), The many faces of National Security in the Arab World, Houndsmills, MacMillan.

Larrabee, F., Greer, J., Lesser, I. y Zanini, M. (1998), NATO's Mediterranean Initiative. Policy Issues and Dilemmas, Sta. Monica, Cal., RAND.

Lesser, I. (2000), Nato Looks South. New Challenges and New Strategies in the Mediterranean, Santa Monica, Cal., RAND.

Lorca, A. (1995), Co-Desarrollo Econômico, Lisboa, Instituto de Estudos Estratégicos e Internacionais (IEEI).

Lorca, A. y Escribano, G. (1998), Geoeconomía y geopolítica mediterráneas, en Revista de Información Comercial Española, № 759.

Lorca, A., Almansa, A. y Jerch, M. (1996), La compatibilité des frontières 'Sud versus Est', en: Revue des Affaires Européenne, 1996.
Marquina, A. (ed.) (1998), Perceptions mutuelles dans la Méditerranée. Unité et diversité, Madrid, UNISCI (Collection STRADMED).

Maxwell, K. (ed.) (1991), Spanish Foreign and Defense Policy, Boulder, Col., Westview Press.

McNamara, R. (1968), The Essence of Security Nueva York, Harper \& Row.

Piris, A. (2001), ¿Por qué empiezan las guerras?, en Muy Especial Guerra y Paz, No 51, enero/ febrero 2001.

Saaf, A. (1995), Le discours stratégique arabe: Constants et variations, Lisboa, Instituto de Estudos Estratégicos e Internacionais (IEEI), (Cahiers du Lumiar $\mathrm{N}^{\circ} 2$ ).

Schölch, I. (1989), Abschreckung, Sicherheit und Stabilität. Grundsatzprobleme der sicherheitspolitischen Situation in Europa, Baden-Baden, Nomos.

Singer, D.(1958), Threat-perception and the armament-tension dilemma, en The Journal of Conflict Resolution, Vol. 2, $\mathrm{N}^{\mathrm{o}} 1$.

Una Europa Segura en un Mundo Mejor. Estrategia Europea de Seguridad, Bruselas, 12 de diciembre de 2003. <http://ue.eu.int/uedocs/ cmsUpload/031208ESSIIES.pdf>. (Consultado 7-7-2005).

Vasconcelos, A. (1999), Régionalisme et Partenariat Euro-Méditerranéen, en Escribano, G. (1999).

Vasconcelos, A. (Coord.) (2002), A European Strategic Concept for the Mediterranean, Lisboa, Instituto de Estudos Estratégicos e Internacionais (IEEI), (Cahiers du Lumiar $\mathrm{N}^{\circ}$ 9).

Wæver, O., Buzan, B., Kelstrup, M. y Lemaitre, P. (eds.) (1993), Identity, Migration and the New Security Agenda in Europe, Londres, Pinter Publisher.

Zaldivar, C. (1991), Spain in the Quest of Autonomy and Security. The Policies of the Socialist Governments, 1982-1990, en Maxwell (1991). 